\title{
ESFINGOFAUNA (LEPIDOPTERA: SPHINGIDAE) DO PARQUE NACIONAL DO JAÚ E ARREDORES, AMAZONAS, BRASIL
}

\author{
Catarina da Silva MOTTA ${ }^{1}$, Ricardo ANDREAZZE ${ }^{2}$
}

\begin{abstract}
RESUMO - A fauna de esfingídeos (Lepidoptera, Sphingidae) foi estudada no Parque Nacional do Jaú e arredores, Amazonas, Brasil, durante o período de julho de 1993 a junho de 1996, O método empregado para as coletas foi luz mista de mercúrio e luz negra UV montadas sobre um lençol branco em períodos de 12 horas consecutivas. Foram coletados 2362 espécimes de 25 gêneros e 79 espécies, com a predominância de espécies das tribos Dilophonotini (45 espécies), seguida por Sphingini (13 spp.), Macroglossini ( $8 \mathrm{spp}$.), Philampelini (7 spp.) e Ambulycini (6 spp.). Os dados são comparados a outras áreas da Amazônia brasileira: Município de Itacoatiara (AM), Iha de Maracá e Pacaraima (RR). Apresenta-se uma lista das espécies de esfingideos incluindo 16 registros novos para o Estado do Amazonas e igualmente para todas as localidades em que foram coletadas.
\end{abstract}

Palavras-chave: Lepidoptera, Bombycoidea, Sphingidae, Parque Nacional do Jaú, Amazônia brasileira.

Hawkmoths Fauna (Lepidoptera, Sphingidae) of the Parque Nacional do Jaú and Surroundings, State of Amazonas, Brazil.

ABSTRACT - A survey of Sphingidae fauna (Lepidoptera, Sphingidae) were studied at Parque Nacional do Jaú and surroundings, Amazonas, Brazil during july/1993 to june/1996. The collects were made using a mixed mercury vapor lamp and black light UV on a white sheet in periods of 12 consecutive hours. 79 hawkmoths species, 25 genera in 2362 specimens were collected with predominance of the species of tribe Dilophonotini ( 45 species), followed by Sphingini (13), Macroglossini (8), Philampelini (7) and Ambulycini (6). Data are compared with others areas of brazilian Amazonia: municipal district of Itacoatiara (AM), Ilha de Maraca and Pacaraima (RR). It is presented a list of species of hawk moths including 16 new register for the State of Amazonas and equal for all localities were collected.

Key-words: Lepidoptera, Bombycoidea, Sphingidae, Parque Nacional do Jaú, Brazilian Amazon.

\section{INTRODUÇÃO}

Os esfingideos são mariposas, na sua maioria noturnos, com cerca de 200 gêneros e 1200 espécies descritas no mundo (Lemaire \& Minet, 1999). $\mathrm{Na}$ região Neotropical temos 312 espécies conhecidas (Heppner, 1991) e segundo Brown (1996), no Brasil ocorrem 180 espécies. Schreiber
(1978) relatou que para a esfingofauna da Hylaea, que não chega a ser um centro de dispersão por faltarem dados locais suficientes, registram-se 76 bem estabelecidas espécies e 24 outras de um ou dois exemplares. Ainda este autor, que trabalhou com material de museus, afirmou a necessidade de mais coletas exaustivas nesta área onde anteriormente só se coletava na

'Coordenação de Pesquisas em Entomologia (CPEN), Instituto Nacional de Pesquisas da Amazônia (INPA), Caixa Postal 478, 69011-970 Manaus, AM, Brasil. e-mail: motta@inpa.gov.br ${ }^{2}$ Coordenação de Pesquisas em Ecologia (CPEC), Instituto Nacional de Pesquisas da Amazônia (INPA), Projeto Dinâmica Biológica de Fragmentos Florestais (PDBFF), Caixa Postal 478, 69011-970 Manaus, AM, Brasil. Bolsista CNPq. e-mail: andreaze@inpa.gov.br 
calha dos grandes rios.

Estes lepidópteros têm grande importância ecológica pela relação inseto-planta, tanto na atuação das lagartas (herbivoria) quanto dos adultos na polinização (nectarivoria) (Motta, 1993). Nas regiões temperadas, os esfingídeos são bem conhecidos como polinizadores, mas, talvez sejam mais significativos nos trópicos, onde eles são polinizadores de muitas árvores, arbustos e epifitas, principalmente Mimosaceae, Rubiaceae, Cactaceae e Orchidaceae (Haber, 1983).

Para a região amazônica brasileira, em grande parte ainda desconhecida quanto à sua esfingofauna, registraram-se nos últimos 10 anos, várias ocorrências de espécies não antes conhecidas para a área (Motta et al. 1991; Motta et al. 1998). Com a implantação do Plano de Manejo do Parque Nacional do Jaú (PNJ), houve a possibilidade de se conhecer uma área nunca antes amostrada, com excursões periódicas, permitindo assim novos registros (Fundação Vitória Amazônica, 1998).

Foram realizadas coletas em diferentes localidades dentro do Parque Nacional do Jaú, eventualmente em Anavilhanas e arredores. Os dados aqui apresentados são registros de novas localidades para os esfingídeos no Parque Nacional do Jaú e na Estação Ecológica de Anavilhanas, Estado do Amazonas, Brasil.

\section{MATERIAL E MÉTODOS}

O Parque Nacional do Jaú (PNJ) tem uma área de 2.272 .000 ha que está localizada entre as latitudes $1^{\circ} 40^{\prime} \mathrm{e}$ $3^{\circ} 00^{\prime} \mathrm{S}$ e longitudes $61^{\circ} 25^{\prime}$ a $63^{\circ} 50^{\prime} \mathrm{W}$, delimitada pelos rios Negro, Jaú e Carabinani, no Municipio de Novo Airão (limite sul do Parque) e pelos rios Unini e Pauini, no Municipio de Barcelos (limite norte), no Estado do Amazonas (Fig.1).

Dentro dos limites do PNJ, 14 localidades foram amostradas em períodos completos de 12 horas e apenas uma localidade foi registro ocasional (Paunini). Nas áreas externas ao Parque, duas coletas (3 noites) foram no arquipélago de Anavilhanas, Município de Novo Airão, pois houve problemas com o motor do barco (em viagens de ida ao PNJ) onde aproveitou-se para coletar estes insetos e duas nas proximidades limites do parque com o rio Negro. Em todas essas localidades, os turnos de coletas foram completos.

As coletas foram realizadas, em geral, nas últimas noites da Lua Minguante e durante a Lua Nova, quando as noites são mais escuras, perfazendo 93 noites de coletas. Foram usados sempre os mesmos tipos de lâmpadas: uma mista de mercúrio e duas de ultra-violeta (luz negra), uma BL "black light" e outra BLB "black light blue", montadas sobre um lençol branco. O uso das lâmpadas UV visava atrair o maior número de insetos, por este trabalho ser parte do projeto geral sobre o inventário da entomofauna do parque.

Os esfíngideos eram coletados manualmente e mortos por injecão de amoniaco. As coletas foram consecutivas e exaustivas, entre 18:00- 


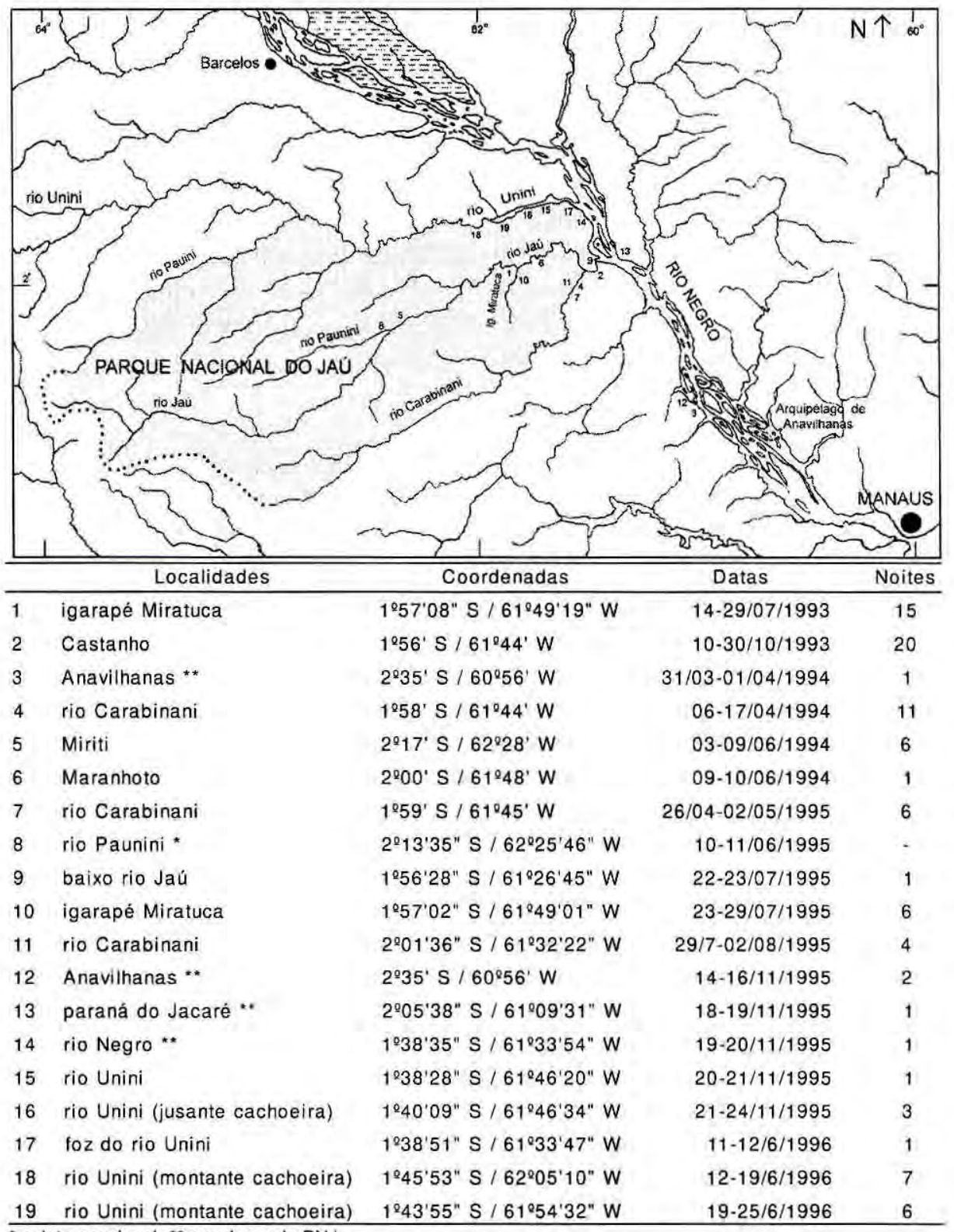

* coleta ocasional ** arredores do PNJ

Figura 1. Localidades amostradas no Parque Nacional do Jaú com as respectivas coordenadas geográficas, período de coleta e número noites. 
06:00h, registrando-se em cada intervalo hora a chegada da mariposa, o sexo e a temperatura local. Quanto às espécies muito comuns apenas marcava-se o indivíduo, com caneta apropriada, e soltava-se, registrando-se seus dados no caderno de campo. Todo material coletado era acondicionado em envelopes entomológicos e foi trabalhado em laboratório no INPA, onde foi montado em esticadores apropriados, identificado, etiquetado definitivamente, recebendo números individuais de Registro/INPA e depositado na Coleção de Invertebrados/ Entomológica do Instituto Nacional de Pesquisas da Amazônia-INPA. A partir dos cadernos de campo foi elaborado um livro de tombo do material coletado e registrado posteriormente no banco de dados da Coleção do INPA.

A classificação e a nomenclatura das espécies dos Sphingidae foi baseada em Kitching \& Cadiou (2000). Para a identificação em nivel de espécie foram utilizados os trabalhos de Rothschild \& Jordan (1903) e D'Abrera (1986).

Nas noites de 21-22 e 22-23/ $10 / 1993$ houve pane no motor gerador sendo levado para conserto em Novo Airão e por isso não houve coletas nestas noites.

\section{RESULTADOS E DISCUSSÃO}

Foram coletados 2362 exemplares de esfingídeos, 79 espécies em 25 gêneros, pela primeira vez coletados nessas localidades amostradas no Parque.
As espécies coletadas no PNJ e arredores, foram as seguintes:

Adhemarius gagarini (Zikán, 1935)

Adhemarius g. gannascus (Stoll, 1780) Adhemarius palmeri (Boisduval, [1875]) Aellopos ceculus (Cramer, 1777) Aleuron carinata (Walker, 1856) Amphimoea walkeri (Boisduval, [1875]) Baniwa yavitensis Lichy, 1981

Callionima inuus (Rothschild \& Jordan, 1903) Callionima nomius (Walker, 1856)

Callionima p. pan (Cramer, 1779) Callionima parce (Fabricius, 1775) Cocytius duponchel (Poey, 1832) Cocytius lucifer Rothschild \& Jordan, 1903 Enyo gorgon (Cramer, 1777)

Enyo l. lugubris (Linnaeus, 1771) Enyo ocypete (Linnaeus, 1758)

Erinnyis a. alope (Drury, 1773)

Erinnyis crameri (Schaus, 1898)

Erinnyis domingonis (Butler, 1875)

Erinnyis e. ello (Linnaeus, 1758)

Erinnyis lassauxii (Boisduval, 1859)

Erinnyis o. obscura (Fabricius, 1775)

Erinnyis oenotrus (Cramer, 1780)

Eumorpha anchemolus (Cramer, 1779) Eumorpha capronnieri (Boisduval, [1875]) Eumorpha fasciatus (Sulzer, 1776) Eumorpha o. obliquus (Rothschild \& Jordan, 1903)

Eumorpha phorbas (Cramer, 1775) Eumorpha s. satellitia (Linnaeus, 1771) Eumorpha v. vitis (Linnaeus, 1758) Eupyrrhoglossum sagra (Poey, 1832) Eupyrrhoglossum venustum Rothschild \& Jordan, 1910

Hemeroplanes ornatus Rothschild, 1894 Hemeroplanes triptolemus (Cramer, 1779) Isognathus excelsior (Boisduval, [1875]) Isognathus leachii (Swainson, 1823) Isognathus menechus (Boisduval, [1875]) 
Isognathus m. mossi Clark, 1919

Isognathus occidentalis Clark, 1929 Isognathus rimosa (Grote, 1865) Isognathus scyron (Cramer, 1780) Isognathus swainsonii Felder \& Felder, 1862 Madoryx plutonius (Hübner, [1819])) Manduca brunalba (Clark, 1929) Manduca leucospila (Rothschild \& Jordan, 1903)

Manduca d. dalica (Kirby, 1877) Manduca diffissa tropicalis (Rothschild \& Jordan, 1903) Manduca florestan (Stoll, 1782) Manduca h. hannibal (Cramer, 1779) Manduca l. lefeburii (Guerin, 1844) Manduca lucetius (Cramer, 1780) Manduca sexta paphus (Cramer, 1779) Neococytius cluentius (Cramer, 1775) Nyceryx coffeae (Walker, 1856) Nyceryx stuarti (Rothschild, 1894) Oryba achemenides (Cramer, 1779) Oryba kadeni (Schaufuss, 1870) Pachylia darceta Druce, 1881 Pachylia ficus (Linnaeus, 1758) Pachylia s. syces (Hübner, [1819]) Pachylioides resumens (Walker, 1856) Perigonia jamaicensis Rothschild, 1894 Perigonia l. lusca (Fabricius, 1777) Perigonia pallida Rothschild \& Jordan, 1903 Perigonia pittieri Lichy, 1962

Protambulyx eurycles (HerrichSchäffer, [1854])

Protambulyx goeldii Rothschild \& Jordan, 1903

Protambulyx strigilis (Linnaeus, 1771) Pseudosphinx tetrio (Linnaeus, 1771) Unzela j. japyx (Cramer, 1776) Unzela p. pronoe (Druce, 1894) Xylophanes amadis (Stoll, 1782) Xylophanes anubus (Cramer, 1777) Xylophanes chiron nechus (Cramer, 1777)
Xylophanes loelia (Druce, 1878)

Xylophanes rufescens (Rothschild, 1894) Xylophanes schausi serenus Rothschild \& Jordan, 1910 Xylophanes t. tersa (Linnaeus, 1771) Xylophanes t. thyelia (Linnaeus, 1758)

Deste total 1567 encontram-se depositados na Coleção Zoológica do INPA (Col, de Invertebrados - Insecta), sob os números de registro: 11001-11007 $(\mathrm{n}=7), 11044-12588$ $(\mathrm{n}=1545), 12801 \quad(\mathrm{n}=1), 14752-14755$ $(n=4), 14759-14765(n=7), 14808$ $14809(n=2) ; 2$ exemplares foram para exposição (Casa da Ciência do INPA), 48 encontram-se na Universidade do Amazonas, Laboratório de Zoologia (Cocytius duponchel com pseudoescorpiões foréticos) e 748 foram marcados e soltos.

Em Macroglossinae registrou-se a maior ocorrência de espécies, 60 spp. (Tab. 1); em ordem decrescente, por tribos: Dilophonotini (45 spp.), Philampelini (7 spp.) e Macroglossini (8 spp.). Embora em Sphinginae tenha-se coletado menos espécies $(\mathrm{n}=13)$ o número de exemplares de Cocytius foi o segundo mais alto. Em Smerinthinae obteve-se 6 spp.

Das 79 espécies coletadas (Tab. 2), 17 apresentaram apenas um espécime: Enyo gorgon, Erinnyis lassauxii, Eumorpha obliquus, Eumorpha vitis, Eupyrrhoglossum sagra, Hemeroplanes triptolemus, Manduca dalica, Manduca lefeburii, Manduca sexta, Nyceryx stuarti, Pachylia syces, Perigonia jamaicensis, Perigonia pittieri, Unzela japyx, Unzela pronoe, Xylophanes anubus e Baniwa yavitensis antes considerada 
endêmica do Estado do Amazonas na Venezuela. Desta espécie, coletou-se o único exemplar fêmea conhecido até o presente (Motta \& Soares, 1997).

Ainda, do total de espécies coletadas, 16 são registros novos para o Estado do Amazonas: Amphimoea walkeri, Cocytius lucifer, Eumorpha o. obliquus, Hemeroplanes ornatus, Isognathus occidentalis, Madoryx plutonius, Manduca leucospila, Nyceryx coffaeae, Pachylia s. syces, Perigonia jamaicensis, Perigonia pallida, Perigonia pittieri, Protambulyx goeldii, Unzela $p$. pronoe, Xylophanes rufescens e

Tabela 1. Sphingidae coletados no Parque Nacional do Jaú, Municipios de Novo Airão e Barcelos, AM entre 07/1993 e 06/1996 (M=machos, $F=$ fêmeas).

\begin{tabular}{|c|c|c|c|c|c|c|c|c|}
\hline \multicolumn{2}{|c|}{ Subfamilias } & Tribos & Subtribos & Gêneros & spp. & M & $\mathrm{F}$ & EXS. \\
\hline \multicolumn{2}{|c|}{ Smerinthinae } & \multirow{2}{*}{\multicolumn{2}{|c|}{ Ambulycini }} & Adhemarius & 3 & 190 & 21 & 211 \\
\hline 418 & (6 spp.) & & & Protambulyx & 3 & 185 & 22 & 207 \\
\hline \multicolumn{2}{|c|}{ Sphinginae } & Sphingini & & Amphimoea & 1 & 5 & 1 & 6 \\
\hline \multirow[t]{3}{*}{554} & (13 spp.) & & & Cocytius & 2 & 354 & 122 & 476 \\
\hline & & & & Manduca & 9 & 30 & 4 & 34 \\
\hline & & & & Neococytius & 1 & 25 & 13 & 38 \\
\hline \multicolumn{2}{|c|}{ Macroglossinae } & Dilophonotini & Dilophonotina & Aellopos & 1 & 1 & 1 & 2 \\
\hline \multirow[t]{20}{*}{1177} & (60 spp.) & 1097 & 1097 & Aleuron & 1 & 4 & - & 4 \\
\hline & & & & Baniwa & 1 & - & 1 & 1 \\
\hline & & & & Callionima & 4 & 75 & 9 & 84 \\
\hline & & & & Enyo & 3 & 61 & 28 & 89 \\
\hline & & & & Erinnyis & 7 & 282 & 204 & 486 \\
\hline & & & & Fupyrhoglossu- & 2 & 47 & 1 & 48 \\
\hline & & & & Hemeroplanes & 2 & 3 & 1 & 4 \\
\hline & & & & Isognathus & 8 & 103 & 10 & 113 \\
\hline & & & & Madoryx & 1 & 10 & 1 & 11 \\
\hline & & & & Nyceryx & 2 & 3 & - & 3 \\
\hline & & & & Oryba & 2 & 4 & - & 4 \\
\hline & & & & Pachylia & 3 & 74 & 25 & 99 \\
\hline & & & & Pachylioides & 1 & 17 & 12 & 29 \\
\hline & & & & Perigonia & 4 & 58 & 8 & 66 \\
\hline & & & & Pseudosphinx & 1 & 38 & 14 & 52 \\
\hline & & & & Unzela & 2 & 1 & 1 & 2 \\
\hline & & Philampelini & & Eumorpha & 7 & 63 & 17 & 80 \\
\hline & & 80 & & & & & & \\
\hline & & Macroglossini & Choerocampina & Xylophanes & 8 & 163 & 50 & 213 \\
\hline & & 213 & 213 & & & & & \\
\hline \multicolumn{2}{|c|}{ TOTAL } & & & 25 & 79 & 1796 & 566 & 2362 \\
\hline
\end{tabular}


Xylophanes schausi serenus.

Das 9 espécies conhecidas como endêmicas (Schreiber, 1978), 2 foram capturadas: Isognathus $m$. mossi (3 exs.) e Nyceryx stuarti com apenas 1 exemplar.

Do total coletado, 10 espécies foram mais abundantes (Tab. 3). Essas espécies também foram já registradas em Roraima e Itacoatiara (Motta et al. 1991; Motta et al. 1998).

A espécie Cocytius duponchel a única coletada em todas as localidades amostradas do Parque foi bastante representativa, provavelmente pela quantidade de plantas hospedeiras (Annonaceae) tanto na área de floresta nativa quanto nas áreas já perturbadas com pequenos cultivos de frutíferas desta família (Motta, 1993).

\section{Comparação com outras áreas da Amazônia brasileira}

Comparando-se as espécies coletadas no PNJ com as de Itacoatiara, percebe-se alguns dados semelhantes quanto à proporção de machos e fềmeas. No PNJ o número de machos capturados no lençol foi cerca de 3 vezes mais do que fêmeas (1796/566), proporção essa semelhante ao observado anteriormente em Itacoatiara.

As duas espécies mais abundantes no Parque Nacional do Jaú, Cocytius duponchel e Erinnyis e. ello também foram abundantes tanto em Roraima quanto em Itacoatiara, sendo que nesta, as do gênero Erinnyis Hübner, [1819] tiveram maior representatividade. No $\mathrm{PNJ}$, constatouse que Erinnyis também teve grande ocorrência, talvez pelas áreas de coleta escolhidas para as amostragens que eram próximas à areas já antes ocupadas por moradores do Parque e também pela proximidade das roças de macaxeira e mandioca.

Entretanto, apesar da aparente alteração empreendida pelas comunidades do Jaú, o ambiente permanece praticamente intacto. Neste levantamento, nota-se que algumas espécies, indicadoras de área perturbada não foram capturadas em quantidade no parque. Xylophanes tersa é uma espécie muito comum em ambientes alterados, onde frequentemente crescem as plantas alimento da lagarta e foi mais representada em Itacoatiara, o mesmo não ocorrendo nas áreas do PNJ.

Outra espécie a ser mencionada é a Baniwa yavitensis que foi capturada na área do igarapé Miratuca, justamente em uma antiga roça de macaxeira.

Todas as ocorrências únicas de espécies, assinaladas dentro da área do Parque, ilustram a riqueza de espécies existente que deve ser estudada. Espécies consideradas "raras" em outras áreas podem deixar de serem a partir da realização de levantamentos faunisticos onde haja disponibilidade de tempo e apoio logístico necessário.

Observando-se o mapa do Parque tem-se também uma idéia da abrangência deste levantamento em relação à área total. Estes estudos que foram iniciais quanto à entomofauna do Parque, localizaram-se basicamente nas partes baixas dos seus 3 rios formadores, que são justamente as áreas mais ocupadas pelos ribeirinhos. No entanto, as áreas investigadas são 
Iิ Tabela 2. Esfingídeos coletados no Parque Nacional do Jaú e arredores, no período de 07/1993 a 06/1996. M=machos, F=fêmeas, TT=total. Os números das localidades correspondem aos da figura 1 .

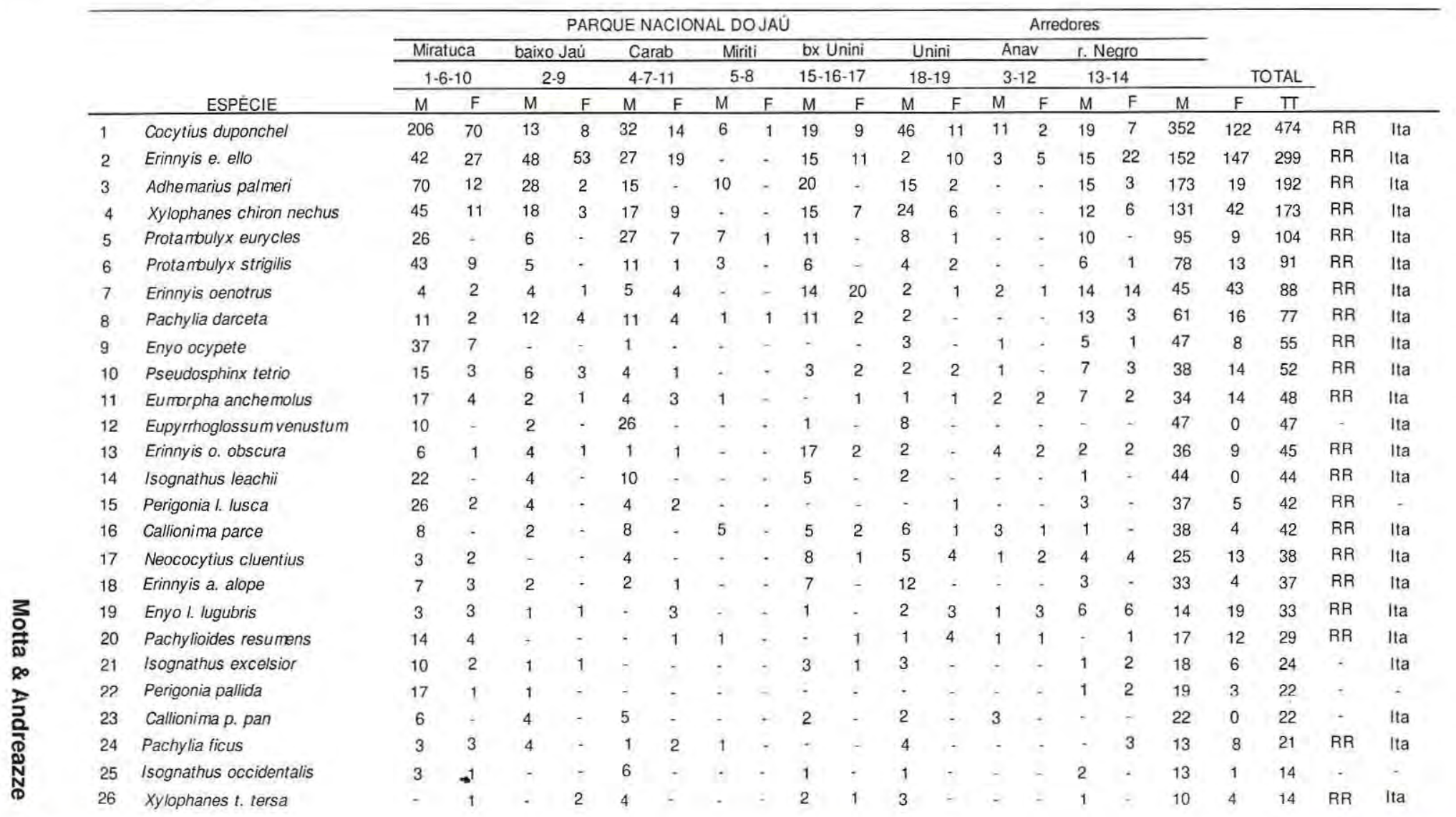


Tabela 2 - Continuação

27 Manduca lucetius

28 Isognathus menechus

29 Adhemarius g. gannascus

30 Eumorpha capronnieri

31 Protambulyx goeldii

32 Callionima nomius

33 Madoryx plutonius

34 Eumorpha phorbas

35 Xylophanes $t$. thyelia

36 Callionima inuus

37 Manduca h, hannibal

38 Erinnyis do mingonis

39 Erinnyis crameri

40 Adhemarius gagarini

41 Eumorphas. satellitia

42 Isognathus rimosa

43 Amphimoea walkeri

44 Isognathus swainsonii

45 Xylophanes amadis

46 Aleuron carinata

47 Xylophanes rufescens

48 Xylophanes loelia

49 Manduca brunalba

50 Xylophanes schausi serenus

51 Isognathus m mossi

52 Hemeroplanes ornatus

53 Aellopos ceculus

54 Isognathus scyron

\begin{tabular}{|c|c|c|c|c|c|c|c|c|c|c|c|c|c|c|c|c|c|c|c|c|c|}
\hline 8 & 1 & - & - & 3 & - & $=$ & - & - & - & - & - & - & - & 1 & & - & 12 & 1 & 13 & - & Ita \\
\hline 3 & - & . & - & 7 & - & - & - & 3 & - & 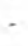 & - & - & - & - & & - & 13 & 0 & 13 & . & Ita+ \\
\hline 4 & . & 5 & - & - & - & - & 1 & 1 & - & - & - & - & - & . & & 1 & 10 & 2 & 12 & RR & Ita \\
\hline 4 & $=$ & 1 & - & 3 & - & . & - & - & 1 & - & - & - & - & & & - & 11 & 1 & 12 & - & Ita \\
\hline 2 & - & - & , & 7 & - & 2 & - & - & . & 1 & $=$ & - & - & & & - & 12 & 0 & 12 & - & - \\
\hline 7 & - & 2 & - & - & + & - & - & - & - & - & - & - & 1 & . & & 1 & 9 & 2 & 1 & RR & Ita \\
\hline 3 & . & 1 & - & 1 & - & 1 & - & - & - & 4 & 1 & . & . & . & & . & 10 & 1 & 11 & - & - \\
\hline 4 & 1 & 3 & - & 1 & - & . & - & - & - & - & . & 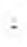 & - & . & & - & 8 & 1 & 9 & . & Ita \\
\hline 4 & - & 1 & $=$ & $=$ & - & - & - & 1 & - & 3 & - & - & - & . & & - & 9 & 0 & 9 & - & Itat \\
\hline 1 & 1 & - & . & . & - & - & . & 2 & 1 & 2 & 1 & - & . & & & - & 6 & 3 & 9 & RR & - \\
\hline - & - & - & - & . & - & - & . & - & . & 1 & - & 6 & 1 & . & & 1 & 7 & 2 & 9 & RR & Ita \\
\hline 4 & - & 1 & , & . & - & . & - & 2 & . & . & - & - & - & & & . & 8 & 0 & 8 & RR & Ita \\
\hline - & 1 & - & - & 2 & - & - & - & 3 & - & - & . & - & - & 2 & & - & 7 & 1 & 8 & RR & Ita \\
\hline 5 & - & - & - & 2 & - & - & - & - & - & - & - & . & . & & $=$ & - & 7 & 0 & 7 & - & Ita \\
\hline 2 & . & - & . & 4 & 1 & - & - & - & - & . & - & - & . & & , & - & 6 & 1 & 7 & RR & Ita+ \\
\hline 1 & - & 2 & $=$ & 3 & - & . & - & - & - & $=$ & - & $=$ & - & & 1 & - & 7 & 0 & 7 & - & Ita \\
\hline 3 & . & - & - & 2 & - & - & - & . & 1 & . & - & - & . & & - & - & 5 & 1 & 6 & $=$ & - \\
\hline 3 & - & - & - & 1 & $=$ & 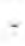 & - & 2 & - & . & - & . & . & & - & - & 6 & 0 & 6 & RR & Ita \\
\hline 3 & 2 & - & . & , & - & - & - & - & - & - & . & - & - & . & - & - & 3 & 2 & 5 & - & Ita \\
\hline 2 & - & - & - & 2 & - & . & - & - & - & - & - & - & . & . & & . & 4 & 0 & 4 & RR & - \\
\hline 1 & 1 & - & . & 1 & - & . & - & 1 & - & - & - & - & - & &. & - & 3 & 1 & 4 & . & - \\
\hline- & - & - & - & 1 & $=$ & - & - & - & - & 3 & - & . & - & & - & . & 4 & 0 & 4 & - & Ita \\
\hline 2 & - & - & - & 1 & - & - & - & - & . & - & - & - & - & . & & . & 3 & 0 & 3 & - & Ita \\
\hline 1 & - & - & . & 1 & 1 & . & - & - & - & . & - & - & . & & & - & 2 & 1 & 3 & - & - \\
\hline . & 1 & - & - & . & - & - & . & c & $i$ & - & - & - & . & & & 1 & 0 & 3 & 3 & $=$ & Ita \\
\hline , & - & 1 & 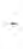 & 1 & 1 & - & . & - & + & . & - & - & & & & - & 2 & 1 & 3 & - & - \\
\hline 1 & 1 & - & . & , & - & - & . & - & - & . & . & - & 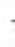 & & . & - & 1 & 1 & 2 & RR & Ita \\
\hline 1 & . & . & . & - & - & . & . & . & $=$ & 1 & - & - & . & & . & - & 2 & 0 & 2 & RR & Ita \\
\hline
\end{tabular}


Tabela 2 - Continuação

\begin{tabular}{|c|c|c|c|c|c|c|c|c|c|c|c|c|c|c|c|c|c|c|c|c|c|c|}
\hline 55 & Manduca florestan & 1 & - & 1 & - & - & - & - & - & - & - & - & - & - & - & - & - & 2 & 0 & 2 & RR & - \\
\hline 56 & Oryba kadeni & 1 & - & - & - & - & - & - & - & - & - & - & - & 1 & - & - & - & 2 & 0 & 2 & RR & Ita \\
\hline 57 & Manduca leucospila & - & - & 1 & 1 & - & - & - & - & - & - & - & - & - & - & - & - & 1 & 1 & 2 & - & - \\
\hline 58 & Nyceryx coffaeae & - & - & 1 & - & - & - & - & - & - & - & 1 & - & - & - & - & $\cdot$ & 2 & 0 & 2 & - & $\cdot$ \\
\hline 59 & Cocytius lucifer & - & - & $\cdot$ & $\cdot$ & 2 & $\cdot$ & - & - & - & - & - & - & - & - & $\cdot$ & $\cdot$ & 2 & 0 & 2 & - & $=$ \\
\hline 60 & Oryba achemenides & - & - & - & $=$ & 1 & - & - & - & - & - & - & $\cdot$ & - & $\cdot$ & 1 & - & 2 & 0 & 2 & RR & - \\
\hline 61 & Eumorpha fasciatus & - & - & - & - & - & - & - & $\cdot$ & 1 & . & 1 & - & - & - & $\cdot$ & - & 2 & 0 & 2 & RR & Ita \\
\hline 62 & Manduca diffissa tropicalis & - & - & - & - & - & - & - & - & - & - & - & - & 2 & - & - & - & 2 & 0 & 2 & RR & Ita \\
\hline 63 & Baniwa yavitensis & - & 1 & - & - & - & - & - & - & - & - & $\cdot$ & - & - & - & - & - & 0 & 1 & 1 & - & - \\
\hline 64 & Eumorpha o. obliquus & 1 & - & $=$ & - & $=$ & - & $=$ & - & - & - & - & - & - & - & - & - & 1 & 0 & 1 & - & - \\
\hline 65 & Manduca I. lefeburii & 1 & - & - & - & - & - & - & - & - & - & - & - & - & - & - & - & 1 & 0 & 1 & - & Ita \\
\hline 66 & Nyceryx stuarti & 1 & - & $\cdot$ & - & $\cdot$ & - & - & $\cdot$ & - & - & - & $\cdot$ & - & - & - & - & 1 & 0 & 1 & RR & - \\
\hline 67 & Xylophanes anubus & 1 & - & - & - & . & - & - & - & - & - & - & $\cdot$ & - & - & - & $\cdot$ & 1 & 0 & 1 & - & Ita+ \\
\hline 68 & Hemeroplanes triptolemus & - & - & 1 & $\cdot$ & - & - & - & - & - & - & - & - & - & - & - & - & 1 & 0 & 1 & - & Ita+ \\
\hline 69 & Unzela p. pronoe & - & $\cdot$ & 1 & - & - & , & - & $\cdot$ & - & $\cdot$ & - & $\cdot$ & - & - & - & $\cdot$ & 1 & 0 & 1 & - & $\cdot$ \\
\hline 70 & Unzela j. japyx & - & - & - & 1 & - & - & - & - & - & , & - & - & - & - & - & - & 0 & 1 & 1 & RR & - \\
\hline 71 & Manduca d. dalica & - & $\cdot$ & - & - & 1 & - & - & - & - & - & - & - & - & - & - & - & 1 & 0 & 1 & - & Ita \\
\hline 72 & Pachylia s. syces & - & - & . & - & - & 1 & - & - & - & $\cdot$ & - & - & - & - & - & - & 0 & 1 & 1 & - & - \\
\hline 73 & Eupyrrhoglossum sagra & - & - & - & - & - & - & - & 1 & $\cdot$ & - & $\cdot$ & - & - & - & - & 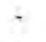 & 0 & 1 & 1 & RR & - \\
\hline 74 & Erinnyis lassauxii & - & - & - & $\cdot$ & - & - & - & - & 1 & - & - & - & - & - & - & - & 1 & 0 & 1 & RR & Ita \\
\hline 75 & Eumorphav. vitis & - & - & - & - & - & - & - & $\cdot$ & 1 & . & - & - & - & - & - & . & 1 & 0 & 1 & - & Ita \\
\hline 76 & Manduca sexta & - & - & - & - & - & - & - & $\cdot$ & 1 & - & - & - & . & - & $\cdot$ & - & 1 & 0 & 1 & RR & Ita \\
\hline 77 & Enyo gorgon & - & - & - & $\cdot$ & - & - & - & - & - & - & - & $\cdot$ & $\cdot$ & 1 & - & - & 0 & 1 & 1 & RR & Ita \\
\hline 78 & Perigonia jamaicensis & . & - & - & $\cdot$ & - & $\cdot$ & - & $\cdot$ & - & - & - & 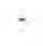 & - & - & 1 & - & 1 & 0 & 1 & - & - \\
\hline 79 & Perigonia pittieri & - & - & - & $\cdot$ & - & $\cdot$ & + & , & - & - & - & - & - & - & 1 & + & 1 & 0 & 1 & - & - \\
\hline \multicolumn{2}{|c|}{ Total de Individuos } & 729 & 180 & 193 & 82 & 272 & 76 & 38 & 5 & 185 & 64 & 177 & 51 & 42 & 22 & 160 & 86 & 1796 & 566 & 2362 & & \\
\hline \multicolumn{2}{|c|}{ Total de Espécies } & \multicolumn{2}{|c|}{58} & \multicolumn{2}{|c|}{37} & \multicolumn{2}{|c|}{46} & \multicolumn{2}{|c|}{13} & \multicolumn{2}{|c|}{37} & \multicolumn{2}{|c|}{34} & \multicolumn{2}{|c|}{17} & \multicolumn{2}{|c|}{37} & \multicolumn{2}{|c|}{79} & & 43 & 54 \\
\hline
\end{tabular}

RR=Espécies de Roraima (Motta et al.1991); Ita=Espécies de ltacoatiara (Motta et al. 1998); Ita+= Espécies a serem acrescentadas para ltacoatiara (dados ainda inéditos). 
Tabela 3. Abundância relativa entre as 10 espécies de Sphingidae mais ocorrentes e as outras, no PNJ e arredores no periodo de 07/1993 a 06/1996.

\begin{tabular}{llcccccc}
\hline & ESPÉCIES & Machos & $\%$ & Fêmeas & $\%$ & Total & $\%$ \\
\hline 1 & Cocytius duponchel & 352 & 19,60 & 122 & 21,55 & 474 & 20,07 \\
2 & Erinnyis e. ello & 152 & 8,46 & 147 & 25,97 & 299 & 12,66 \\
3 & Adhemarius palmeri & 173 & 9,63 & 19 & 3,36 & 192 & 8,13 \\
4 & Xylophanes chiron nechus & 131 & 7,29 & 42 & 7,42 & 173 & 7,32 \\
5 & Protambulyx eurycles & 95 & 5,29 & 9 & 1,59 & 104 & 4,40 \\
6 & Protambulyx strigilis & 78 & 4,34 & 13 & 2,30 & 91 & 3,85 \\
7 & Erinnyis oenotrus & 45 & 2,51 & 43 & 7,60 & 88 & 3,73 \\
8 & Pachylia darceta & 61 & 3,40 & 16 & 2,83 & 77 & 3,26 \\
9 & Enyo ocypete & 47 & 2,62 & 8 & 1,41 & 55 & 2,33 \\
10 & Pseudosphinx tetrio & 38 & 2,12 & 14 & 2,47 & 52 & 2,20 \\
\hline & Total & 1172 & 65,26 & 433 & 76,50 & 1605 & 67,95 \\
\hline & Outras spp. & 624 & 34,74 & 133 & 23,50 & 757 & 32,05 \\
\hline & Total Geral & 1796 & 100 & 566 & 100 & 2362 & 100 \\
\hline
\end{tabular}

pouco alteradas por eles e distantes uma das outras (pontuais) em relação à área do PNJ.

\section{CONCLUSÃO}

O levantamento faunístico realizado no Parque Nacional do Jaú, proporcionou o conhecimento das espécies na região em localidades nunca estudadas bem como o enriquecimento da coleção de esfingídeos na Coleção Entomológica do INPA. Os resultados, em relação à ambrangência da área do PNJ, ilustram a necessidade da continuidade de estudos na região. Como a biologia da maior parte das espécies ainda é desconhecida, os Sphingidae têm no Jaú uma área de preservação e santuário para a ciência, sendo a identificação das espécies apenas o inicio para se prosseguir nas investigações.

\section{AGRADECIMENTOS}

À Fundação Vitória Amazônica pela iniciativa e atenções nas primeiras coletas no PNJ; aos moradores do PNJ pelas atenções e calorosa acolhida; às tripulações dos barcos Pium, Amanai I e Amanaí II, do INPA, pelo apoio dado nas várias excursões; especialmente ao Comandante Raimundo Carvalho e Antonio Torquato do Pium, pela presteza e dedicação com que sempre nos destacou; aos técnicos e pesquisadores do INPA (CPEN; CPEC; CPCS), companheiros nas coletas e pelos valiosos auxilios recebidos.

\section{Bibliografia citada}

Brown Jr., K.S. 1996. Diversity of Brazilian Lepidoptera: history of study, methods for measurement, and use as indicator for genetic, specific and system richness. In: Bicudo, C.E. de M; Menezes, N.A. (eds.) 
1996. Biodiversity in Brazil: a first approach. São Paulo, CNPq: 221-253.

D'Abrera, B. 1986. Sphingidae Mundi. Hawk Moths of the World. Ed. Classey E.W. Ltd., Oxon, 266 p.

Fundação Vitória Amazônica. 1998. A Gênese de um Plano de Manejo. O caso do Parque Nacional do Jaú. Fundação Vitória Amazônica, Manaus, FVA 113 p.

Haber, W.A. 1983. Checklist of Sphingidae. In: JANZEN, D.H., 1983. Costa Rican Natural History. University of Chicago Press, Chicago, 816p.

Heppner, J.B. 1991. Faunal Regions and the Diversity of Lepidoptera. Tropical Lepidoptera 2, Supplement 1,85p.

Kitching, I.J.; Cadiou, J.M. 2000. Hawkmoths of the world: an annotated and illustrated revisionary checklist (Lepidoptera: Sphingidae). Cornell University Press, Ithaca, $227 \mathrm{p}$.

Lemaire, C.; Minet, J. 1999. The Bombycoidea and their Relatives. In: Kristensen, N.P. (ed.) Lepidoptera, Moths and Butterflies. Vol.1: Evolution, Systematics, and Biogeography. Part 35 In: Handbook of Zoology Vol. IV Arthropoda: Insecta. Walter de Gruyter, Berlin-New York.

Motta, C.S. 1993. Mariposas esfingideas (Lepidoptera,Sphingidae) da Amazônia Brasileira e o Meio Ambiente. In: Ferreira, E.J.G., Santos, G.M., Leão, E.L.M. \& Oliveira. L.A. (eds.) Bases Cientificas para Estratégias de Preservação e Desenvolvimento da Amazônia - Fatos e Perspectivas, INPA. Vol.II:141-146.

Motta, C.S.; Soares, A. 1997. Ocorrência de Baniwa yavitensis Lichy, 1961 (Lepidoptera, Sphingidae) na Amazônia brasileira. Acta Amazonica, 27(4): 303307.

Motta, C.S.; Ferreira, R.L.M.; Aguiar, N.O. 1991. Sobre a esfingofauna da ilha de Maracá e da serra de Pacaraima, Roraima
(Lepidoptera, Sphingidae). Acta Amazonica, 21(Único): 319-324.

Motta, C.S.; Aguilera-Peralta, F.J.; Andreazze, R. 1998. Aspectos da Esfingofauna (Lepidoptera: Sphingidae), em área de terra firme, no Estado do Amazonas, Brasil. Acta Amazonica, 28(1): 75-92.

Rothschild, W.; Jordan, K. 1903. A revision on the Lepidoptera family Sphingidae. Novitates Zool. IX, suppl., London, DXXXV 67 pls., 972p.

Schreiber, H. 1978. Dispersal Centres of Sphingidae (Lepidoptera) in the Neotropical Region. Biogeographica X :1-195, Junk \& The Hague ed., Boston. 